\title{
BILATERAL BREAST CARCINOMA - A STUDY OF PATIENTS ADMITTED IN A UNIVERSITY EMERGENCY HOSPITAL
}

\section{Oana C. VOINEA ${ }^{1 凶}$, Adelina BIRCEANU COROBEA ${ }^{2}$, Ion DANIEL ${ }^{3}$, Adriana E. NICA ${ }^{4}$, Maria SAJIN ${ }^{1}$}

${ }^{1}$ Department of Pathology, University of Medicine and Pharmacy „Carol Davila“, University Emergency Hospital, Bucharest, Romania

${ }^{2}$ Department of Pathology, „Sf. Maria“ Clinical Hospital, Bucharest, Romania

${ }^{3}$ Clinical Department of Surgery III, „Carol Davila“ University of Medicine and Pharmacy, University Emergency Hospital, Bucharest, Romania

${ }^{4}$ Department of Anesthesiology and Intensive Care, Carol Davila" University of Medicine and Pharmacy, University Emergency Hospital, Bucharest, Romania

* All the authors contributed equally to the study

\section{Abstract}

Introduction Breast carcinoma represents the leading cause of oncologic mortality among women worldwide. Bilateral breast carcinoma is a distinct pathologic entity, with particular prognostic and therapeutic implications. Objective The objective of the study was to determine the histologic and immunohistochemical characteristics of patients with bilateral breast carcinoma admitted in a university emergency hospital from Bucharest, Romania.

Materials and methods We analyzed breast carcinoma specimens registered as bilateral in the Department of Pathology of the University Emergency Hospital, Bucharest, Romania, between 2015-2017, and studied their histologic and molecular features in the clinical context obtained from our hospital database.

Results All the analyzed patients were postmenopausal women. The extent of time between the diagnosis of

\section{RÉsumÉ}

Le carcinome bilatéral du sein - une étude des patients admis à un hôpital universitaire d'urgence

Introduction Le carcinome du sein représente la principale cause de mortalité oncologique chez les femmes du monde entier. Le carcinome bilatéral du sein est une entité pathologique distincte, ayant des implications pronostiques et thérapeutiques particulières.

Objectif Le but de cette étude est de déterminer les caractéristiques histologiques et immunohistochimiques des patients atteints d'un carcinome bilatéral du sein, admis dans un hôpital universitaire d'urgence.

Matériels et méthodes Nous avons analysé les échantillons de carcinome du sein enregistrés en tant que bilatérales au Département de Pathologie de l'Hopital Universitaire d'Urgence de Bucarest, Roumanie, entre 2014 et 2017, et étudié leurs caractéristiques 
the first and second tumor varied in metachronous cases from 8 months to 7 years. In the majority of cases, the bilateral breast lesions shared histologic, grading and hormone expression similarities.

Conclusions Bilateral breast carcinoma is a rare entity that needs more histologic parameters to be defined as primary or metastatic.

Keywords: breast carcinoma, hormonal receptors, synchronous breast tumor, mastectomy.

\section{INTRODUCTION}

Breast carcinoma represents the oncologic condition with the greatest morbidity and mortality among women worldwide, representing a quarter of all cancers in women ${ }^{1}$. Considering the great frequency of this disease, many studies were made for improving the accuracy of diagnostic, solutions for treatment, all aiming for a better survival and quality of life. Considering the latest guideline for the therapeutic management of breast carcinoma regarding the intrinsic molecular subtypes, treatment became much more personalized, and hormonotherapy and monoclonal antibody are usual in today's oncologic practice ${ }^{2}$. Considering all this progress, there are still a great number of deaths caused by breast carcinoma. The reasons vary from late presentation to medical service, the absence (in some countries, e.g. Romania) of a national screening program of breast malignancies ${ }^{3}$ and also because in some particular type of cancers, such as bilateral breast carcinoma, no standardized therapeutic protocol is available ${ }^{4}$

Bilateral breast cancer is defined by the World Health Organization (WHO) as primary malignant lesions developed in each breast. They are considered synchronous if a contralateral breast carcinoma is diagnosed within 3 months after diagnosing the first tumor and are labeled as metachronous if more time passed ${ }^{5}$. Its frequency varies in clinical reports from $1.4 \%$ to $11.8 \%$. Few statistics quantified how important a primary breast cancer is a risk factor for developing a contralateral breast cancer $^{10}$, considering the side effects of neoadjuvant treatment, but its contribution is undoubtable ${ }^{11}$. Women that already have breast cancer are at two-to six-fold risk for developing another breast cancer in the contralateral breast, compared with the risk of developing a primary breast cancer in the general population ${ }^{8}$. histologiques et moléculaires dans le contexte clinique obtenue à partir de notre base de données hospitalière. Résultats Tous les patients analysés étaient des femmes ménopausées. Le délai entre le diagnostic de la première et de la deuxième tumeur variait de 8 mois à 7 ans dans les cas métachrones.

Conclusions Le carcinome mammaire bilatéral est une entité rare qui nécessite plus de paramètres histologiques pour être définie comme primaire ou métastatique.

Mots-clés: cancer du sein, récepteurs hormonaux, tumeur du sein synchrone, mastectomie.

The objective of the Study was to determine the histologic and immunohistochemical characteristics of patients with bilateral breast carcinoma admitted in a university emergency hospital.

\section{Materials AND MEthods}

We collected our information for this retrospective series of cases from the Pathology Department of the University Emergency Hospital, Bucharest, Romania. All registered breast lesions from the $1^{\text {st }}$ of January 2015 to the $31^{\text {st }}$ of December 2017 in our department were pathologically evaluated. Patients' clinical data and medical history were obtained from our hospital database.

Pathological evaluation included the macroscopic registered data about tumor's dimensions and proximity to the surgical resection margins. The histologic specimens were fixed in 10\% formalin and embalmed in paraffin blocks, 3 -micrometers sections cut and usual stained hematoxylin and eosin. The WHO $4^{\text {th }}$ edition guidelines for breast pathology was used for assessing the histotype, grade of differentiation (according to Elston Ellis grading system) and TNM classification.

For each case, slides that contained well-preserved and representative tumor mass were chosen for immunohistochemistry tests. We used estrogen receptor antibody (SP1 clone, BioCare), progesterone receptor antibody (SP2 clone, BioCare) for the hormonal profile, Ki 67 (MIB-1, BioCare) as the nuclear antigen associated with cell proliferation and cerB-2/ HER2 oncoprotein (rabbit monoclonal antibody). Only nuclear staining was counted for ER, PR, and $\mathrm{Ki}-67$; for HER2neu, the membranous staining was classified according to ASCO-CAP guidelines 2016. When recommended, in situ hybridization tests were completed in another laboratory, and we registered the final result (HER 2 amplified or not). 
The evaluation of prognostic factors made possible to further classify tumors according to the St. Gallen consensus (2011) into molecular surrogate sub-types. Thereby, Luminal A cancers were hormone positive, HER2 negative and had a low proliferative index $(\mathrm{Ki} 67<14 \%)$, Luminal B cancers were also hormone positive, but with a high mitotic index (Ki $67>14 \%$ ) and/or HER2 positive. Non-luminal cancers were either HER2 enriched (did not express ER or PR) or triple negative (nor ER, PR or HER2 were positive).

All clinical data and histopathologic results were processed and analyzed using Microsoft Office Excel 2018. All the patients included in the study signed an informed consent according to the Helsinki agreement and the current national laws.

\section{Results}

A number of 354 breast tumors obtained by core biopsy, lumpectomy, quadrantectomies, modified mastectomy or mastectomy with lymph node dissection were evaluated, with benign diagnostic (most of them fibroadenomas and fibrocystic disease) or malignancies. 248 cases were carcinomas, most of them of no special type, about a quarter were of lobular type and sporadic cases of rare histotypes were also noted (Table 1).

Considering the surrogate classification of molecular subtypes, the most frequent model met was Luminal B, followed by Luminal A (Figure 1). Few cases were of non-luminal type.

We selected the patients who were surgically treated for malignancy in both breasts. During the evaluated time, we found 6 patients, representing $4.83 \%$ of the total investigated cases. A synthetic
Table 1. Histotype and grade frequency of breast carcinomas.

\begin{tabular}{ccccc}
\hline Histotype & 2015 & 2016 & 2017 & Total \\
\hline Mixed & 1 & 0 & 0 & 1 \\
\hline NST G1 & 6 & 9 & 11 & 26 \\
\hline NST G2 & 41 & 43 & 60 & 144 \\
\hline NST G3 & 7 & 5 & 8 & 20 \\
\hline ILC G1 & 1 & 0 & 0 & 1 \\
\hline ILC G2 & 11 & 8 & 6 & 25 \\
\hline ILC G3 & 1 & 4 & 3 & 8 \\
\hline Mucinous Carcinoma & 0 & 4 & 1 & 5 \\
\hline $\begin{array}{c}\text { Adenosquamous } \\
\text { Carcinoma }\end{array}$ & 1 & 1 & 1 & 3 \\
\hline Metaplastic Carcinoma & 0 & 1 & 0 & 1 \\
\hline $\begin{array}{c}\text { NST with neuroendo- } \\
\text { crine features }\end{array}$ & 1 & 1 & 0 & 2 \\
\hline Medullary Carcinoma & 2 & 2 & 2 & 6 \\
\hline $\begin{array}{c}\text { Phyllodes Tumor } \\
\text { Papillary Carcinoma }\end{array}$ & 0 & 0 & 2 & 2 \\
\hline Total & 74 & 79 & 1 & 4 \\
\hline
\end{tabular}

presentation is easy to appreciate in Table 2. Analyzed separately from synchronous breast carcinomas, in metachronous cases the median time-lapse until the diagnosis of the second breast malignancy was 9.9 months, with a range of variation from 8 months to 7 years.

In this brief analysis, we will underline the most important particularities of synchronous and metachronous breast carcinomas separately.

For synchronous bilateral breast carcinoma, in one case of three, the lesion was of low dimensions, diagnosed on a routine mammography evaluation and

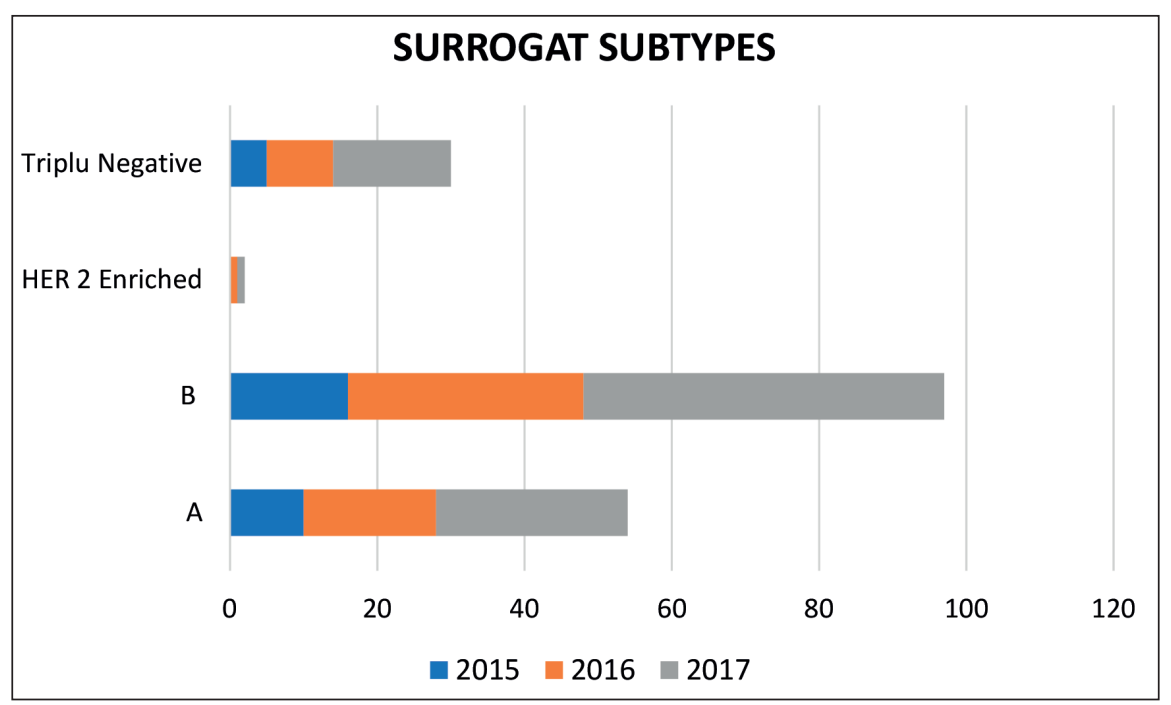

Figure 1. The number of cases according to surrogate classification. 
Table 2. The first and second tumor variables

\begin{tabular}{|c|c|}
\hline First Breast & Second Breast \\
\hline \multicolumn{2}{|c|}{ Age at Diagnostic } \\
\hline 66 & 67 \\
\hline 54 & 61 \\
\hline 60 & 62 \\
\hline 84 & 84 \\
\hline 48 & 48 \\
\hline 50 & 50 \\
\hline \multicolumn{2}{|c|}{ Tumoral Stage } \\
\hline IV & IV \\
\hline 0 & IIA \\
\hline IV & IV \\
\hline IIIB & biopsy \\
\hline IIB & IA \\
\hline Ia & Ia \\
\hline \multicolumn{2}{|c|}{ Grade and Histotype } \\
\hline NST G2 & NST G2 \\
\hline IDC & Adenosquamous \\
\hline NST G1 & NST G3 \\
\hline NST G2 & NST G2 \\
\hline NST G2 & NST G1 \\
\hline NST G1 & NST G1 \\
\hline
\end{tabular}

quantified as BI-RADS 5, with no clinical symptoms. The chosen surgical treatment was mastectomy with lymph node dissection. The histopathological evaluation revealed lesions in each breast, of $1.8 \mathrm{~cm}$ (right) and $0.5 \mathrm{~cm}$ (left), with a similar histotype (NST) and grade of differentiation (G1). On examined slides, no in situ component, tumoral embolism or perineural tumoral invasion were observed. After immunohistochemical stains, we classified it in Luminal A subtype, both tumors expressed both ER and PR, had a low $(10 \%)$ ki-67 and did not express HER2. None of the excised lymph nodes presented metastasis.

Another patient is quite similar to the first presented case, but with some individualities. In the right breast, there were multiple NST tumors, each of them with a moderate grade of differentiation (G2), none of them larger than $1.8 \mathrm{~cm}$. Also, 1 of 8 examined lymph nodes presented metastasis. The contralateral breast had a single well differentiated (G1) tumor of NST histotype, with no affected locoregional lymph node. According to prognostic factors immunohistochemically acquired, we classified all tumors from both breasts of luminal A type. The elected surgical treatment was also bilateral mastectomy with lymph node dissections.

In contrast with the anterior presented cases, the third one had a stage IIIB breast bilateral carcinoma. Considering the emergency profile of our hospital, the patient presented an ulcerated bleeding breast mass. The clinical and imaging examination evaluated both breasts and noticed malignant features bilaterally. An emergency mastectomy was performed, and a biopsy was obtained from the contralateral breast. The diagnosis in both, mastectomy specimen and biopsy, was NST G2, of Luminal A profile (Figure 2). No in situ component was found, but tumoral emboli, perineural invasion and skin ulceration by tumoral proliferation were noticed (Figure $3 \mathrm{~A}$ and $3 \mathrm{~B}$ ). No additional pathologic information about lymph node invasion was obtained.

The metachronous cases we found are about to be debated considering their primitive or metastatic origin. In 2 of 3 patients, they were in advanced stages of disease (stage IV), with distant metastases (with pulmonary propensity in both cases). We did not find significant differences in histotype (in both patients, bilateral NST), or in molecular profile (both Luminal $B$ and HER2 negative) but the grade of differentiation was not the same, in the second diagnosed tumor being higher (Table 2). No in situ component was identified, in first or second diagnosed breast affected by cancer. A particular aspect was the benign associated lesions that we found. Fibroadenomas coexisted with fibrocystic breast disease and NST carcinoma in both breasts (Figure 3C and 3D).

A single case presented with a history of in situ carcinoma treated with mastectomy and local radiotherapy. After 7 years, in the contralateral breast, an adenosquamous carcinoma developed.

From the clinical point of view, all cases were postmenopausal women, 5 of 6 patients were overweight and hypertensive; half of them had imbalanced thyroid function; 2 of them associated usual gynecological pathology (cervicitis, uterine leiomyomas) and half of them came from the rural area. The median diameter of tumors was $2.3 \mathrm{~cm}$, with a variation ranging from 0.8 to $3.4 \mathrm{~cm}$.

\section{Discussion}

To distinguish the synchronous from metachronous bilateral breast cancer, we used the $\mathrm{WHO} 4^{\text {th }}$ edition guideline cutoff of 3 months interval between diagnosing the first and the contralateral breast tumor. Even in the literature, no consensus about the outcome of bilateral breast carcinoma compared to unilateral ones, most of them suggest a poor survival, in particular for women affected by synchronous breast carcinomas ${ }^{12-15}$.

Considering the molecular subtype, the majority of our cases were of luminal type, meaning that were expressing hormonal receptors. The higher prevalence of hormonal receptor positive bilateral breast 


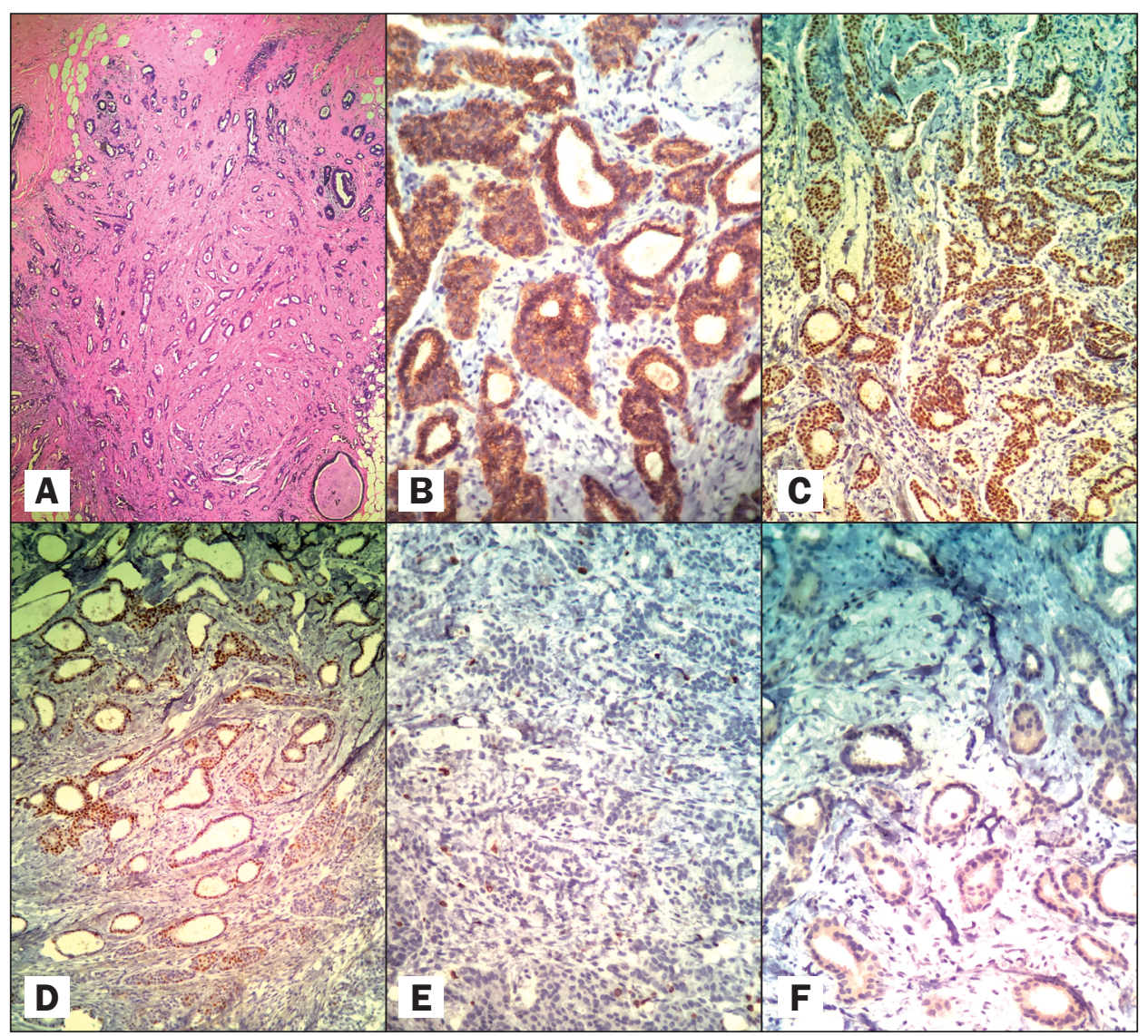

Fig. 2. NST breast carcinoma of Luminal A subtype. A. HE, 4X; B. E-caderine, 20X; C. ER 10X, 98\% positivity; D.PR 10X,70\% positivity; E. Ki67, 20X, 10\% positivity; F. HER2 neu, 20X, 1+.

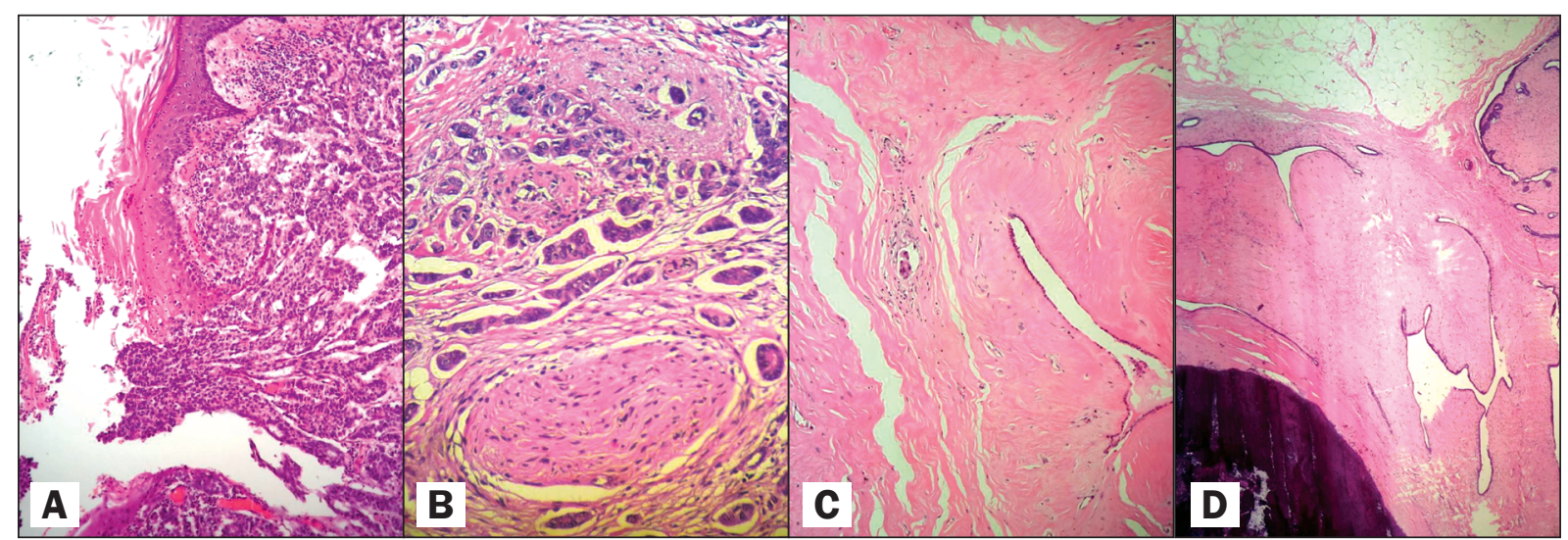

Fig. 3. Invasive NST T4d carcinoma. A. Skin invasion, HE 4X; B. Perineural Invasion HE, 20X, C. tumoral emboli near to a fibroadenoma, HE, 10X; D. calcifications in fibroadenoma, HE, 4X

carcinomas was similar to other studies ${ }^{16}$. In the literature, it is noticed that the elevated risk of a second malignancy of breast cancer survivals is related to hormonal receptor status ${ }^{17,18}$. The similarities in histologic appearance and hormone receptor positivity in the same patient could be an argument for the hypothesis that tumors arise from a common circumstance and their subtypes are predetermined in the early stage of breast cancer development $t^{19,20}$.
A more problematic aspect was to distinguish a bilateral breast cancer from a contralateral metastatic spread. The second tumor, in the absence of local, regional or distant metastasis, it is compatible with a second primary cancer ${ }^{9,21}$. Otherwise, in common histologic terms, it is impossible to do this differentiation $^{22,23}$. We searched the literature and we found various criteria ${ }^{24-26}$, none being approved as a golden standard. Despite innovative diagnostic methods, 
such as cDNA microarray-based CGH, the most used is the Chaudaty et al proposal, elaborated in $1984^{21}$. This study stipulates that a bilateral breast carcinoma, comparative to a metastatic one, riches the following criteria: a). the presence of in situ changes in the contralateral tumor b). histologic differences between the first tumor and the contralateral one c). the degree of histologic differentiation of the tumor in the second breast is distinctly greater than that of the lesion in the first breast and d). no evidence of local, regional or distant metastasis from cancer in the ipsilateral breast.

Our presented cases do not fulfill all the Chaudaty criteria, but clinical and histological aspects made us think that metastatic disease is less probable. In the synchronous bilateral breast carcinoma, considering the incipient disease, the absence of vascular and perineural tumor invasion, we presumed that tumors are both primaries. We believe the same for the patient with a history of in situ ductal carcinoma and contralateral adenosquamous carcinoma. The problematic aspects are in patients with stage 4 disease, where it is difficult to establish the primary or metastatic nature of the lesion. Considering the severity of disease, in this point of medical knowledge, the distinction would bring no clinical utility.

An interesting point represents the association in one case of bilateral fibroadenoma, that, according to Dupont and al, could be a risk factor for malignancies ${ }^{27}$.

A clinical aspect that is further to be investigated is the strong correlation between hypertension and thyroid function. Several articles observed a connection between benign or malignant breast disease and thyroid dysfunction ${ }^{28.30}$, and the majority of cases correlated with a lower thyroid function ${ }^{31}$. Considering hypertension, there are studies about this correlation, some of them with astonishing results, considering that the risk of a hypertensive woman for developing a breast carcinoma is up to $15 \%$ higher compared to general population ${ }^{32,33}$.

\section{Conclusions}

Although bilateral breast carcinomas are rare cases, their early diagnosis is mandatory. In non-metastatic diseases, we have found important similarities in tumors' histology and hormonal profile in both breasts, so, an accurate distinction between primary or metastatic disease is not possible using only histopathologic examinations. Also, the longtime risk of metachronous breast cancer confirms the importance of mammographic follow up of ipsi- and contralateral breast of patients already treated of breast cancer ${ }^{34}$.

\section{Compliance with Ethics Requirements:}

„The authors declare no conflict of interest regarding this article"

"The authors declare that all the procedures and experiments of this study respect the ethical standards in the Helsinki Declaration of 1975, as revised in 2008(5), as well as the national law. Informed consent was obtained from all the patients included in the study"

No funding for this study

\section{RefEREnCES}

1. Ferlay J, Soerjomataram I, Ervik M, et al. GLOBOCAN 2012 v1.0, Cancer Incidence and Mortality Worldwide: IARC CancerBase No. 11 Lyon, France: International Agency for Research on Cancer; 2013. Available from: http://globocan.iarc.fr (Accessed on June 10, 2018).

2. Untch M, Gerber B, Harbeck N et al. 13th St. Gallen International Breast Cancer Conference 2013: Primary Therapy of Early Breast Cancer Evidence, Controversies, Consensus - Opinion of a German Team of Experts (Zurich 2013), Breast Care (Basel) 2013; 8(3): 221-229.

3. Marmot MG, Altman DG, Cameron DA, et al. The benefits and harms of breast cancer screening: an independent review. British Journal of Cancer 2013;108(11):2205-2240.

4. Reimer T. Management of rare histological types of breast tumours. Breast Care (Basel) 2008;3:190-196.

5. Hartman M, Czene K, Reilly M, et al. Incidence and prognosis of synchronous and metachronous bilateral breast cancer. Journal of Clinical Oncology 2007;25:4210-4216.

6. Michowitz M, Noy S, Lazebnik N, Aladjem D. Bilateral breast cancer. Journal of Surgical Oncology 1985, 30: 109-112.

7. Gogas J, Markopoulos C, Skandalakis P, Gogas H. Bilateral breast cancer. The American Surgeon 1993; 59(11):733-735.

8. Chen Y, Thompson W, Semenciw R, Mao Y. Epidemiology of contralateral breast cancer. Cancer Epidemiolgy, Biomarkers EO Prevention 1999; 8(10): 855-861.

9. Donovan AJ. Bilateral breast cancer. The Surgical Clinics of North America 1990; 70(5):1141- 1149.

10. Roder D, De Silva P, Zorbas H, et al. Survival from synchronous bilateral breast cancer: the Experience of surgeons participating in the breast audit of the Society of Breast Surgeons of Australia and New Zealand. Asian Pacific Journal of Cancer Prevention 2012; 13(4): 1413-1418.

11. Hooning MJ, Aleman BM, Hauptmann M, et al. Roles of radiotherapy and chemotherapy in the development of contralateral breast cancer. Journal of Clinical Oncology 2008, 26(34):5561-5568.

12. Levi F, Randimbison L, Te V, La Vecchia C. Prognosis of bilateral synchronous breast cancer in Vaud, Switzerland. Breast 2003;12(2):89-91.

13. Heron DE, Komarnicky LT, Hyslop T, Schwartz GF, Mansfield CM. Bilateral breast carcinoma: risk factors and outcomes for patients with synchronous and metachronous disease. Cancer 2000;88(12):2739-2750.

14. Jobsen JJ, van der Palen J, Ong F, Meerwaldt JH. Synchronous, bilateral breast cancer: prognostic value and incidence. Breast 2003;12(2):83-88.

15. Vichapat V, Garmo H, Holmberg L, et al. Patterns of metastasis in women with metachronous contralateral breast cancer. British Journal of Cancer 2012; 107(2):221-223. 
16. Diaz R, Munarriz B, Santaballa A, Palomar L, Montalar J. Synchronous and metachronous bilateral breast cancer: a long- term single-institution experience. Medical Oncology 2012, 29(1):16-24.

17. Liu J, Jiang W, Mao K, et al. Elevated risks of subsequent endometrial cancer development among breast cancer survivors with different hormone receptor status: a SEER analysis. Breast Cancer Res Treat 2015; 150:439-445.

18. Schonfeld SJ, Curtis RE, Anderson WF. et al. The risk of a second primary lung cancer after a first invasive breast cancer according to estrogen receptor status. Cancer Causes E Control 2012; 23:1721-1728

19. Bu-Ali H, Solh M, Kapur A, Mittal V. Receptor characteristics of the second tumor in synchronous versus metachronous breast cancer. The American Surgeon 2008; 74(8):702705.

20. Huo D, Melkonian S, Rathouz PJ, Khramtsov A, Olopade OI. Concordance in histological and biological parameters between first and second primary breast cancers. Cancer 2011, 117(5):907-915.

21. Chaudary MA, Millis RR, Hoskins EO, et al. Bilateral primary breast cancer: a prospective study of disease incidence. British Journal of Surgery 1984; 71(9):711-714.

22. Robinson E, Rennert G, Bar-Deroma R, Dori DL, Neugut AI . The pattern of diagnosis of a second primary tumor in the breast. Breast Cancer Research and Treatment 1993; 25(3):211-215.

23. Younes M, Lane M, Miller C, Laucirica R. Stratified multivariate analysis of prognostic markers in breast cancer: a preliminary report. Anticancer Research 1997, 17: 1383-1390.

24. Broët P, Rochefordière A, Scholl SM, et al. Contralateral breast cancer: metastasis or second primary? Bulletin $d u$ Cancer 1996; 83(10):870-876
25. Jobsen JJ, van der Palen J, Ong F, Riemersma S, Struikmans H. Bilateral breast cancer, synchronous and metachronous; differences and outcome. Breast Cancer Research and Treatment 2015, 153(2):277-283

26. Bernstein JL, Thompson WD, Risch N, Holfort TR. The genetic epidemiology of second primary breast cancer. American Journal of Epidemiology 1992; 136(8): 937-948.

27. Dupont WD, Page DL, Parl FF, et al. Long term risk of breast cancer in women with fibroadenoma. New England Journal of Medicine 1994; 331(1):10-15.

28. Adamopoulos DA, Vassilaros S, Kapolla N, Papadiamantis J, Georgiakodis F, Michalakis A. Thyroid disease in patients with benign and malignant mastopathy. Cancer 1986; 57:125-128.

29. Lemaire, M, Baugnet-Mahieu, L. Thyroid function in women with breast cancer. European Journal of Cancer Clinical Oncology 1986; 22:301-307.

30. Paraschiv B, Dediu G, Iancu A, Bratu O, Diaconu C. Superior vena cava syndrome. Arch Balk Med Union 2017;52(1):39-43

31. Mittra, I, Hayward, JL. Hypothalamic-pituitary-thyroid axis in breast cancer. Lancet 1974; 1:885-889.

32. Han H, Guo W, Shi W, et al. Hypertension and breast cancer risk: a systematic review and meta-analysis. Scientific Report 2017; 7:44877.

33. Diaconu C. Comorbidities of hypertensive patients: are there differences between men and women? Arch Balk Med Union 2016;51(3):307-310.

34. Robertson C, Kumar S, Ragupathy A, et al. Surveillance mammography for detecting ipsilateral breast tumor recurrence and metachronous contralateral breast cancer: a systematic review. European Radiology 2011; 21(12): 2482-2491. 